\title{
In Vitro Susceptibility of Candida Species to Four Antifungal Agents Assessed by the Reference Broth Microdilution Method
}

\author{
Fahriye Eksi, Efgan Dogan Gayyurhan, and Iclal Balci \\ Department of Medical Microbiology, Faculty of Medicine, Gaziantep University and Universite Bulvari, 27310 Gaziantep, Turkey \\ Correspondence should be addressed to Fahriye Eksi; eksi@gantep.edu.tr
}

Received 22 July 2013; Accepted 8 September 2013

Academic Editors: A. Joshi and A. Ouwehand

Copyright (C) 2013 Fahriye Eksi et al. This is an open access article distributed under the Creative Commons Attribution License, which permits unrestricted use, distribution, and reproduction in any medium, provided the original work is properly cited.

\begin{abstract}
This study was performed to determine the distribution of Candida species isolated from the blood cultures of the patients hospitalized in our hospital and to investigate their antifungal susceptibility. Candida strains were identified at species level by using classical methods and API ID 32C (bioMerieux, France) identification kits. The susceptibility of the strains to amphotericin $\mathrm{B}$, fluconazole, voriconazole, and caspofungin was evaluated by using the reference broth microdilution method in document M27A3 of the Clinical and Laboratory Standards Institute. Of the 111 Candida strains isolated, $47.7 \%$ were identified as C. albicans and $52.3 \%$ as non-albicans Candida strains. The MIC ranges were $0.03-1 \mu \mathrm{g} / \mathrm{mL}$ for amphotericin B, $0.125-\geq 64 \mu \mathrm{g} / \mathrm{mL}$ for fluconazole, $0.03-16 \mu \mathrm{g} / \mathrm{mL}$ for voriconazole, and $0.015-0.25 \mu \mathrm{g} / \mathrm{mL}$ for caspofungin. All Candida strains were susceptible to amphotericin B and caspofungin. $10.8 \%$ isolates were resistant to fluconazole and $8.1 \%$ isolates were dose-dependent susceptible. While $0.9 \%$ isolate was resistant to voriconazole, $0.9 \%$ isolate was dose-dependent susceptible. In our study, C. albicans and C. parapsilosis were the most frequently encountered agents of candidemia and it was detected that voriconazole with a low resistance rate might also be used with confidence in the treatment of infections occurring with these agents, primarily besides amphotericin B and caspofungin.
\end{abstract}

\section{Introduction}

The species of fungi included in genus Candida are normally microorganisms which are found in the flora of the human skin and mucosa. Oral colonization by Candida albicans has been reported at $17.7 \%$ in the healthy population. Among hospitalized patients, oral carriage of Candida albicans rises to $40.6 \%$. The infections which occur with pathogenic fungi, Candida species in particular, are both common and increasing in frequency [1]. Candidemia and invasive candidiasis are a major cause of nosocomial infections, linked to a number of risk factors such as prior antimicrobial therapy, venous and urinary catheters, intensive care unit admission, parenteral nutrition, major surgery, and immunosuppressive therapies $[2,3]$. Although $C$. albicans is the most frequent agents of candidemia, it has shown an increasing rate of nonalbicans Candida species over the past decades. Compared with incidences from the 1980s, a larger proportion of Candida bloodstream infections are now caused by Candida glabrata in the United States and by Candida parapsilosis and Candida tropicalis in European, Canadian, and Latin
American hospitals. The most important reasons for the increase in candidemia depending on nonalbicans Candida strains are the prophylactic and empirical administration of antifungals, particularly of azole drugs due to their easy use and to their large spectrum [4]. In many studies, both the incidence and agent spectrum of candidemia agents were reported [5].

Even though the number of systemically effective antifungal agents used in the treatment of Candida infections is not very large, several antifungals may be used including amphotericin B, azole group of drugs, flucytosine, and echinocandins [6]. The choice of initial antifungal agent for the empirical treatment of a suspected invasive Candida infection is also difficult. Development of resistance to the antifungal agents used in Candida infections is a serious problem. The use of fluconazole prophylaxis over the last two decades is believed to have led to an increase in nonalbicans species with reduced fluconazole susceptibility [7]. Therefore, there is an increasing need for in vitro antifungal susceptibility testing to choose the most appropriate and effective antifungal agent. For this purpose, the Clinical and Laboratory Standards Institute (CLSI) 
issued reports in order to identify the standard methods in both yeasts and molds. Of them, M27-A3 Reference Method for Broth Dilution Antifungal Susceptibility Testing of Yeasts, Approved Standard, is the microdilution method developed to determine the susceptibility of yeasts to antifungal agents $[8]$.

This study aimed to identify the Candida species isolated from the blood cultures of the patients hospitalized in various clinics, particularly the intensive care clinic of our hospital, at species level and to determine their susceptibility to amphotericin $\mathrm{B}$, fluconazole, voriconazole, and caspofungin by using the standard broth microdilution method.

\section{Materials and Methods}

This study was approved by the Ethics Committee of the Faculty of Medicine at Gaziantep University. The 111 Candida isolates collected from blood cultures from patients hospitalized in various clinics of the Medical Faculty Hospital at Gaziantep University between March 2008 and January 2009 were evaluated. Of the blood cultures, 39 (35.1\%) were obtained from patients hospitalized in the intensive care clinic, $15(13.5 \%)$ in the podiatry clinic, $15(13.5 \%)$ in the pediatric oncology clinic, $9(8.1 \%)$ in the internal medicine clinic, and the remaining $33(29.8 \%)$ in the other clinics. The germ tube test, microscopic appearance on Cornmeal Tween 80 agar, determination of the colour of colonies on Candida ID 2 agar-a chromogenic medium-and API ID 32C (bioMerieux, France) identification kit were used for the identification of yeast strains.

Antifungal susceptibility testing was performed using the broth microdilution method. The Clinical and Laboratory Standards Institute developed and published an approved reference method for the broth microdilution testing (CLSI document M27-A3) of Candida species [8]. The standard powders of fluconazole (Sigma), amphotericin B (Sigma), voriconazole (Pfizer), and caspofungin (Merck) were used as antifungals. Distilled water was used as a solvent for fluconazole and caspofungin, whereas DMSO (dimethylsulphoxide) (Sigma) was used as a solvent for water-insoluble amphotericin B and voriconazole. The stock solutions were prepared at the rate of $1280 \mu \mathrm{g} / \mathrm{mL}$ for fluconazole, $1600 \mu \mathrm{g} / \mathrm{mL}$ for amphotericin B, $1600 \mu \mathrm{g} / \mathrm{mL}$ for voriconazole, and $1600 \mu \mathrm{g} /$ $\mathrm{mL}$ for caspofungin. For the susceptibility test, RPMI 1640 (with glutamine, bicarbonate-free, and containing phenol red as the $\mathrm{pH}$ indicator) (Sigma) was used as a medium. The final concentrations were in the range $64-0.125 \mu \mathrm{g} / \mathrm{mL}$ for fluconazole, $16-0.03 \mu \mathrm{g} / \mathrm{mL}$ for amphotericin B and voriconazole, and $8-0.015 \mu \mathrm{g} / \mathrm{mL}$ for caspofungin. Each Candida strain was studied twice for fluconazole, amphotericin B, voriconazole, and caspofungin. The results were evaluated 24 hours later for caspofungin and 48 hours later for fluconazole, amphotericin $\mathrm{B}$, and voriconazole. For amphotericin B, the MIC endpoint was defined as the lowest drug concentration that resulted in a reduction in growth by $90 \%$ or more, compared with that of a drug-free growth control well. For fluconazole, the MIC endpoint was defined as a $50 \%$ reduction in optical density. For caspofungin, the endpoint was defined according
TABLE 1: Distribution of Candida species isolated from blood cultures.

\begin{tabular}{lcc}
\hline Species & $N$ & $\%$ \\
\hline C. albicans & 53 & 47.8 \\
C. parapsilosis & 41 & 36.9 \\
C. krusei & 6 & 5.4 \\
C. tropicalis & 3 & 2.7 \\
C. glabrata & 3 & 2.7 \\
C. kefyr & 2 & 1.8 \\
C. lusitaniae & 2 & 1.8 \\
C. famata & 1 & 0.9 \\
\hline Total & 111 & 100 \\
\hline
\end{tabular}

to the recently published reports that analyzed influences of methodological variables on susceptibility testing of caspofungin against Candida species, though an interpretive cutoff value is not yet available for this echinocandin [9]. Therefore, the endpoint is given as the concentration of the drug in the assay at which $50 \%$ of growth control was observed. The in vitro susceptibility tests of the isolated Candida species were interpreted considering the guide prepared by the CLSI [10]. Although no resistance extreme was specified for amphotericin B by the CLSI, the strains with a MIC value of $>1$ are accepted as resistant [8]. Since C. krusei is an intrinsically fluconazole-resistant Candida species, no resistance extremes were used in the interpretation of the MIC values obtained for C. krusei [10]. Quality control was ensured by testing the CLSI-recommended strains C. parapsilosis ATCC 22019 and C. krusei ATCC 6258 [10].

Statistical significance was determined by chi-square analysis (Epi Info, version 6).

\section{Results}

Of the 111 Candida strains isolated, 53 (47.7\%) were identified as C. albicans, $41(36.9 \%)$ as C. parapsilosis, $6(5.4 \%)$ as $C$. krusei, 3 (2.7\%) as C. tropicalis, $3(2.7 \%)$ as C. glabrata, $2(1.8 \%)$ as C. kefyr, and $2(1.8 \%)$ as C. lusitaniae, while one (0.9\%) was identified as C. famata (Table 1). The MIC ranges and $\mathrm{MIC}_{50}$ and $\mathrm{MIC}_{90}$ values of the identified strains according to the antifungal agent are given in Table 2 .

All Candida strains isolated were detected to be susceptible to amphotericin B and caspofungin.

Fluconazole resistance and dose-dependent susceptibility were detected in $3(5.66 \%)$ and $6(11.3 \%)$ of the C. albicans isolates $(n=53)$, respectively, and no resistance was detected for voriconazole.

Of the nonalbicans Candida strains ( $n=58), 9(15.5 \%)$ were found resistant to fluconazole (all C. krusei strains were accepted as resistant), while 3 (5.2\%) were found dosedependent susceptible. Of the 6 C. krusei strains isolated, 4 strains were found to have a MIC value of $\geq 64 \mu \mathrm{g} / \mathrm{mL}$ and 2 strains were found to have a MIC value of $16 \mu \mathrm{g} / \mathrm{mL}$. Since the C. krusei strains were intrinsically resistant to fluconazole, all of them were accepted as resistant to fluconazole regardless of the MIC values determined in vitro. Of the 3 strains that 
TABLE 2: MIC ranges as well as $\mathrm{MIC}_{50}$ and $\mathrm{MIC}_{90}$ values of the identified strains according to the antifungal agent.

\begin{tabular}{|c|c|c|c|c|}
\hline Species $(n)$ & Antifungal agent & MIC range $(\mu \mathrm{g} / \mathrm{mL})$ & $\mathrm{MIC}_{50}(\mu \mathrm{g} / \mathrm{mL})$ & $\mathrm{MIC}_{90}(\mu \mathrm{g} / \mathrm{mL})$ \\
\hline \multirow{4}{*}{ C. albicans (53) } & Fluconazole & $0.25-32$ & 0.5 & 16 \\
\hline & Amphotericin B & $0.03-0.25$ & 0.06 & 0.125 \\
\hline & Voriconazole & $0.03-0.25$ & 0.03 & 0.03 \\
\hline & Caspofungin & $0.015-0.25$ & 0.03 & 0.06 \\
\hline \multirow{4}{*}{ C. parapsilosis (41) } & Fluconazole & $0.25-64$ & 0.5 & 1 \\
\hline & Amphotericin B & $0.03-0.25$ & 0.06 & 0.125 \\
\hline & Voriconazole & $0.03-0.06$ & 0.03 & 0.03 \\
\hline & Caspofungin & $0.06-0.25$ & 0.125 & 0.25 \\
\hline \multirow{4}{*}{ C. tropicalis (3) } & Fluconazole & $0.25-64$ & 1 & 64 \\
\hline & Amphotericin B & $0.125-0.25$ & 0.125 & 0.25 \\
\hline & Voriconazole & $0.03-16$ & 0.06 & 16 \\
\hline & Caspofungin & $0.015-0.06$ & 0.06 & 0.06 \\
\hline \multirow{4}{*}{ C. krusei (6) } & Fluconazole & $16->64$ & 64 & $>64$ \\
\hline & Amphotericin B & $0.25-1$ & 0.25 & 1 \\
\hline & Voriconazole & $0.06-0.5$ & 0.125 & 0.5 \\
\hline & Caspofungin & $0.015-0.25$ & 0.015 & 0.25 \\
\hline \multirow{4}{*}{ C. kefyr (2) } & Fluconazole & $0.25-0.5$ & 0.25 & 0.5 \\
\hline & Amphotericin B & $0.125-0.5$ & 0.125 & 0.5 \\
\hline & Voriconazole & $0.03-0.03$ & 0.03 & 0.03 \\
\hline & Caspofungin & $0.03-0.06$ & 0.03 & 0.06 \\
\hline \multirow{4}{*}{ C. glabrata (3) } & Fluconazole & $16->64$ & 64 & $>64$ \\
\hline & Amphotericin B & $0.125-0.125$ & 0.125 & 0.125 \\
\hline & Voriconazole & $0.25-2$ & 1 & 2 \\
\hline & Caspofungin & $0.015-0.06$ & 0.03 & 0.06 \\
\hline \multirow{4}{*}{ C. lusitaniae (2) } & Fluconazole & $0.5-2$ & 0.5 & 2 \\
\hline & Amphotericin B & $0.125-0.125$ & 0.125 & 0.125 \\
\hline & Voriconazole & 0.03 & 0.03 & 0.03 \\
\hline & Caspofungin & $0.06-0.06$ & 0.06 & 0.06 \\
\hline \multirow{4}{*}{ C. famata (1) } & Fluconazole & 16 & 16 & 16 \\
\hline & Amphotericin B & 0.25 & 0.25 & 0.25 \\
\hline & Voriconazole & 0.25 & 0.25 & 0.25 \\
\hline & Caspofungin & 0.03 & 0.03 & 0.03 \\
\hline
\end{tabular}

were resistant to fluconazole except for C. krusei, one was detected as C. tropicalis and 2 were detected as C. glabrata. Of the 3 strains detected to be dose-dependent susceptible, one was detected as C. glabrata $(\mathrm{MIC}=16 \mu \mathrm{g} / \mathrm{mL})$, one as C. famata $(\mathrm{MIC}=16 \mu \mathrm{g} / \mathrm{mL})$, and one as C. parapsilosis $(\mathrm{MIC}=16 \mu \mathrm{g} / \mathrm{mL})$. No statistically significant difference in fluconazole resistance was found between $C$. albicans and nonalbicans strains $\left(\chi^{2}=2.79, P=0.094\right)$.

Voriconazole resistance was detected in one $(1.72 \%)$ of the nonalbicans Candida strains, while dose-dependent susceptibility was detected in one (1.72\%) of them. C. tropicalis was detected as the strain that was resistant to voriconazole and C. glabrata as the dose-dependent susceptible strain. No statistically significant difference in voriconazole resistance was found between C. albicans and nonalbicans strains $\left(\chi^{2}=\right.$ $0.92, P=0.336)$.

\section{Discussion}

The frequency of invasive mycoses due to opportunistic fungal pathogens has increased significantly over the past two decades. More than 17 different species of Candida have been identified as etiologic agents of bloodstream infections. Approximately 95\% of all Candida bloodstream infections are caused by four species: C. albicans, C. glabrata, C. parapsilosis, and $C$. tropicalis [11]. Of the Candida strains isolated in our study, $47.7 \%$ were identified as C. albicans, $36.9 \%$ as $C$. parapsilosis, $5.4 \%$ as C. krusei, $2.7 \%$ as C. tropicalis, $2.7 \%$ as C. glabrata, and the remaining $4.5 \%$ as C. kefyr, C. famata, and $C$. lusitaniae. These results are compatible with the other research results $[12,13]$. C. albicans almost always ranks first, while the frequency of nonalbicans species varies according to various studies. In many studies performed in the recent years, it has been expressed that there has been an evident change in agents of candidemia and that the rate of candidemia depending on nonalbicans Candida strains has reached approximately 50\% [14]. Although C. albicans was the most frequently observed species in the study that was carried out by Malani et al. [12] and that investigated agents of candidemia in a 12-year period between 1988 and 1999, its proportion decreased from $63 \%$ to $43 \%$. Nevertheless, the incidence of C. glabrata increased to $20 \%$ from $10 \%$, while 
the incidence of C. parapsilosis increased to $18 \%$ from $5 \%$. Cuenca-Estrella et al. [13] identified some 351 Candida strains isolated from the blood samples and reported that they had found C. albicans at the rate of $51 \%, C$. parapsilosis at the rate of $23 \%$, C. tropicalis at the rate of $10 \%$, C. glabrata at the rate of $9 \%$, and $C$. krusei at the rate of $4 \%$. In 2003, Messer et al. [15] examined some 1,397 Candida strains, most of which were isolated from the blood culture, from North America, Europe, and Latin America within the scope of SENTRY Antimicrobial Surveillance Program and they identified $48.7 \%$ of them as C. albicans, $17.3 \%$ of them as $C$. parapsilosis, $17.2 \%$ of them as C. glabrata, $10.9 \%$ of them as $C$. tropicalis, $1.9 \%$ of them as C. krusei, and $4 \%$ of them as other Candida species. C. parapsilosis is notorious for the ability to form biofilms on catheters and other implanted devices, for nosocomial spread by hand carriage, and for persistence in the hospital environment. It is also well known for causing infections in infants and neonates [3].

The increase in fungal infections has prompted an increase in the use of antifungal agents, and in practice the widespread clinical use of these agents has resulted in measurable rates of acquired or innate fungal resistance in Candida species [3]. A new period initiated in the treatment of fungal infections with the discovery of amphotericin B in 1953. Even though amphotericin B is one of the most toxic antimicrobial agents in clinical use, it still qualifies as a standard treatment. Resistance is reported in nonalbicans Candida species, for example, species $C$. lusitaniae and $C$. guilliermondii [16]. Clinical failure to respond to amphotericin B treatment occurs with the nonrecovery of the factors about the immune system of the host rather than with in vitro resistance [17]. Amphotericin B resistance may vary by region, and the resistance profile may also vary at different times in the same region. In our study, the MIC values of the 111 Candida strains under examination were in the range $0.03-1 \mu \mathrm{g} / \mathrm{mL}$ for amphotericin $\mathrm{B}$, and no resistant ( $\geq 2 \mu \mathrm{g} / \mathrm{mL}$ ) strain was encountered. Barchiesi et al. [18] from Italy detected that the MIC values for amphotericin B were in the range $0.03-0.5 \mu \mathrm{g} / \mathrm{mL}$ in 56 Candida strains; Godoy et al. [19] from Latin America detected that the MIC values for amphotericin B were in the range $0.125-1 \mu \mathrm{g} / \mathrm{mL}$ in 103 Candida strains; and Cuenca-Estrella et al. [13] from Spain detected that the MIC values of $351 \mathrm{C}$. albicans strains for amphotericin B were in the range $0.03-0.5 \mu \mathrm{g} / \mathrm{mL}$, and they did not find any amphotericin B resistance, which is parallel with our study. In various studies carried out in our country $[20,21]$, they did not detect any amphotericin B resistance in the Candida strains isolated from the blood cultures either. However, in their study, Kiraz et al. [22] found the MIC range as $0.03-4 \mu \mathrm{g} / \mathrm{mL}$ and the $\mathrm{MIC}_{50}$ and $\mathrm{MIC}_{90}$ values as 0.5 and $1 \mu \mathrm{g} / \mathrm{mL}$ in 300 C. albicans strains, respectively. On the other hand, different results were obtained in the studies performed in various countries. Diekema et al. [23] detected that the MIC values of amphotericin B in 254 Candida strains were in the range $0.25-2 \mu \mathrm{g} / \mathrm{mL}$, while they observed the MIC value as $2 \mu \mathrm{g} / \mathrm{mL}$ in only 7 strains and reported $3 \%$ resistance. Cuenca-Estrella et al. [24] determined the MIC range of amphotericin B as $0.03-2 \mu \mathrm{g} / \mathrm{mL}$ and the rate of resistance as $2.7 \%$ in 514 Candida strains obtained from Spain, whereas they determined the MIC range as $0.06-8 \mu \mathrm{g} / \mathrm{mL}$ and the rate of resistance as $0.86 \%$ in some 230 Candida strains obtained from Argentina.

Today the most frequently used antifungals systemically and locally are the azole group of agents. Of the azoles used systemically, fluconazole is the most frequently used one in yeasts. Among Candida species, C. krusei is intrinsically resistant to fluconazole. Furthermore, the susceptibility of $C$. glabrata strains also varies widely. While some C. glabrata strains are dose dependent and fluconazole susceptible, about $15 \%$ of them display real resistance. Following prolonged fluconazole prophylaxis in patients with AIDS in particular, acquired resistance to fluconazole might develop even in C. albicans isolates [17]. In our study, the MIC values for fluconazole were detected between 0.125 and $64 \mu \mathrm{g} / \mathrm{mL}$ for the 111 Candida strains under examination and fluconazole resistance was $5.66 \%$ in C. albicans strains and $15.5 \%$ in nonalbicans candida strains. The number of dose-dependent susceptible $(16-32 \mu \mathrm{g} / \mathrm{mL})$ isolates was $9(8.1 \%)$, and all $C$. krusei strains were accepted as resistant. Sabatelli et al. [25] detected $6.4 \%$ resistance to fluconazole in 6,595 Candida isolates. Skrodeniene et al. [26] found 14 (15.1\%) of $93 \mathrm{C}$. albicans strains resistant to fluconazole, while Sojakova et al. [27] reported fluconazole resistance as $13 \%$ in 227 Candida isolates. The rates of resistance are reported in a quite wide range in the studies carried out in our country as well. While no resistance was seen at all in some studies [21, 22], Kantarcioglu and Yücel [28] detected 38.8\% resistance in nonalbicans Candida strains, while Kaya et al. [29] detected $68.7 \%$ resistance in $32 \mathrm{C}$. albicans strains isolated from neutropenic patients with malignity and $63.2 \%$ resistance in 106 nonalbicans strains.

Voriconazole is a synthetic triazole derived from fluconazole. As a result of structural changes, its activity of inhibiting the target enzyme lanosterol demethylase increased and its spectrum extended [30]. It is also effective on fluconazoleresistant Candida strains. Nevertheless, a significant number of fluconazole-resistant Candida isolates also become resistant to voriconazole as a result of cross-resistance besides being resistant to ketoconazole and itraconazole [17]. In our study, the MIC range of voriconazole was 0.03$16 \mu \mathrm{g} / \mathrm{mL}$ for the 111 Candida strains evaluated. The number of voriconazole-resistant $(\geq 4 \mu \mathrm{g} / \mathrm{mL})$ Candida strains was $1(1.72 \%)$, whereas one strain $(1.72 \%)$ was dose-dependent susceptible $(2 \mu \mathrm{g} / \mathrm{mL})$. The voriconazole-resistant strain was detected as $C$. tropicalis. Accordingly, no voriconazole resistance was detected for C. albicans strains, while voriconazole resistance was $1.72 \%$ in nonalbicans strains. In the study where Espinel-Ingroff et al. [31] investigated the voriconazole resistance of 90 Candida strains, they did not detect any resistance in $20 \mathrm{C}$. albicans strains, whereas they detected resistance in 3 (4.3\%) of 70 nonalbicans isolates. In addition, although the fluconazole resistance of $C$. albicans strains was $11 / 20(55 \%)$ in this study, no voriconazole resistance was observed [31]. Swinne et al. [16] detected the MIC range of voriconazole as $\leq 0.008-16 \mu \mathrm{g} / \mathrm{mL}$ in 121 nonalbicans Candida strains and reported resistance in 4 of them (3.3\%), whereas Alexander et al. [32] detected the MIC values for voriconazole as $<0.03->64$ for 212 Candida strains and found voriconazole 
resistance in 7 of them (3.3\%). Likewise, Sabatelli et al. [25] found the voriconazole resistance of 6,595 Candida isolates as $3.3 \%$. The results of each of these last three studies are parallel with our study. The rates of resistance quite vary in the studies carried out in our country. Keçeli Özcan et al. [33] detected voriconazole resistance in $2(4.7 \%)$ of 43 nonalbicans Candida strains, whereas Aydin et al. [34] did not detect any voriconazole resistance in 166 Candida strains.

Echinocandins are in general active against various Candida and Aspergillus spp. Their favorable activity against azole and amphotericin B resistant as well as azole and amphotericin B susceptible Candida strains is one of the major advantages of echinocandin use in clinical practice $[17,30]$. Caspofungin and anidulafungin have been approved by the US Food and Drug Administration for the treatment of invasive candidiasis, including candidemia [3]. All of the strains examined in our study were found susceptible to caspofungin ( $\mathrm{MIC} \leq 2 \mu \mathrm{g} / \mathrm{mL}$ ). In their study, Pfaller et al. [35] did not encounter any caspofungin-resistant strains in 8,197 Candida isolates. Likewise, Alexander et al. [32] detected the MIC range of caspofungin as $<0.03-2 \mu \mathrm{g} / \mathrm{mL}$ in 212 Candida strains and they found no caspofungin resistance in any of them.

\section{Conclusions}

Our results also confirm the elevated incidence of bloodstream infections caused by nonalbicans Candida strains. It was concluded that voriconazole with a low resistance rate might also be used with confidence in the treatment of infections occurring with Candida species, primarily besides amphotericin B and caspofungin. Moreover, identification at species level in Candida strains is necessary particularly in terms of detecting C. krusei and the other species that might be resistant to fluconazole.

\section{Conflict of Interests}

The authors have no conflict of interests.

\section{Acknowledgment}

This study was supported with Project no. TF.08.08 by the Unit of Scientific Research Projects at Gaziantep University.

\section{References}

[1] J. Masuoka, "Surface glycans of Candida albicans and other pathogenic fungi: physiological roles, clinical uses and experimental challenges," Clinical Microbiology Reviews, vol. 17, no. 2, pp. 281-310, 2004.

[2] A. Viudes, J. Peman, E. Canton, P. Ubeda, J. L. Lopez-Ribot, and M. Gobernado, "Candidemia at a tertiary-care hospital: eidemiology, treatment, clinical outcome and resk factors for death," European Journal of Clinical Microbiology \& Infectious Diseases, vol. 21, pp. 767-774, 2002.

[3] M. A. Pfaller and D. J. Diekema, "Epidemiology of invasive candidiasis: a persistent public health problem," Clinical Microbiology Reviews, vol. 20, no. 1, pp. 133-163, 2007.
[4] B. Almirante, D. Rodríguez, B. J. Park et al., "Epidemiology and predictors of mortality in cases of Candida bloodstream infection: results from population-based surveillance, Barcelona, Spain, from 2002 to 2003," Journal of Clinical Microbiology, vol. 43, no. 4, pp. 1829-1835, 2005.

[5] P. Sandven, "Epidemiology of candidemia," Revista Iberoamericana de Micologia, vol. 17, no. 3, pp. 73-81, 2000.

[6] G. Maschmeyer and M. Ruhnke, "Update on antifungal treatment of invasive Candida and Aspergillus infections," Mycoses, vol. 47, no. 7, pp. 263-276, 2004.

[7] J. R. Perfect, "Antifungal resistance: the clinical front," Oncology, vol. 18, no. 14, pp. 15-22, 2004.

[8] Clinical and Laboratory Standards Institute (CLSI), Reference Method for Broth Dilution Antifungal Susceptibility Testing of Yeasts; Approved Standard-Third Edition. CLSI Document M27$A 3$, Clinical and Laboratory Standards Institute, Wayne, Penn, USA, 2008.

[9] F. C. Odds, M. Motyl, R. Andrade et al., "Interlaboratory comparison of results of susceptibility testing with caspofungin against Candida and Aspergillus species," Journal of Clinical Microbiology, vol. 42, no. 8, pp. 3475-3482, 2004.

[10] Clinical and Laboratory Standards Institute (CLSI), Reference Method for Broth Dilution Antifungal Susceptibility Testing of Yeasts; Third Informational Supplement. CLSI Document M27S3, Clinical and Laboratory Standards Institute, Wayne, Penn, USA, 2008.

[11] M. A. Pfaller and D. J. Diekema, "Rare and emerging opportunistic fungal pathogens: concern for resistance beyond Candida albicans and Aspergillus fumigatus," Journal of Clinical Microbiology, vol. 42, no. 10, pp. 4419-4431, 2004.

[12] P. N. Malani, S. F. Bradley, R. S. Little, and C. A. Kauffman, "Trends in species causing fungaemia in a tertiary care medical centre over 12 years," Mycoses, vol. 44, no. 11-12, pp. 446-449, 2001.

[13] M. Cuenca-Estrella, D. Rodriguez, B. Almirante et al., "In vitro susceptibilities of bloodstream isolates of Candida species to six antifungal agents: results from a population-based active surveillance programme, Barcelona, Spain, 2002-2003," Journal of Antimicrobial Chemotherapy, vol. 55, no. 2, pp. 194-199, 2005.

[14] H. Richet, P. Roux, C. Des Champs, Y. Esnault, A. Andremont, and French Candidemia Study Group, "Candidemia in French hospitals: incidence rates and characteristics," Clinical Microbiology and Infection, vol. 8, no. 7, pp. 405-412, 2002.

[15] S. A. Messer, R. N. Jones, and T. R. Fritsche, "International surveillance of Candida spp. and Aspergillus spp.: report from the SENTRY Antimicrobial Surveillance Program (2003)," Journal of Clinical Microbiology, vol. 44, no. 5, pp. 1782-1787, 2006.

[16] D. Swinne, M. Watelle, M. Van der Flaes, and N. Nolard, "In vitro activities of voriconazole (UK-109, 496), fluconazole, itraconazole and amphotericin B against 132 non-albicans bloodstream yeast isolates (CANARI study)," Mycoses, vol. 47, no. 5-6, pp. 177-183, 2004.

[17] S. Arikan and J. H. Rex, "Antifungal agents," in Manual of Clinical Microbiology, P. R. Murray, E. J. Baron, M. L. Landry, J. H. Jorgensen, and M. A. Pfaller, Eds., pp. 1949-1960, ASM Press, Washington, DC, USA, 9th edition, 2007.

[18] F. Barchiesi, G. Caggiano, M. Maracci, D. Arzeni, G. Scalise, and M. T. Montagna, "Antifungal susceptibility patterns of yeast isolates causing bloodstream infections," Journal of Antimicrobial Chemotherapy, vol. 51, no. 2, pp. 431-433, 2003.

[19] P. Godoy, I. N. Tiraboschi, L. C. Severo et al., "Species distribution and antifungal susceptibility profile of Candida species 
bloodstream isolates from Latin American hospitals," Memorias do Instituto Oswaldo Cruz, vol. 98, no. 3, pp. 401-405, 2003.

[20] N. Ü. Toprak, S. Erdoğan, C. Çelik, and C. Johansson, "In vitro susceptibility to Amphotericin B and Fluconazole of Candida strains isolated froom blood cultures," Türk Mikrobiyoloji Cemiyeti Dergisi, vol. 33, pp. 252-256, 2003.

[21] G. Yenisehirli, Y. Bulut, and E. Günday, "Antifungal susceptibility of Candida albicans isolates recovered from blood cultures of intensive care unit patients," Ankem Dergisi, vol. 21, no. 3, pp. 146-149, 2007.

[22] N. Kiraz, Z. Erturan, M. Uzun et al., "Susceptibility of 300 Candida albicans strains to amphotericin B, flucytosine, flukonazole ve mikonazole," Klimik Dergisi, vol. 11, no. 3, pp. 116-118, 1998.

[23] D. J. Diekema, S. A. Messer, A. B. Brueggemann et al., "Epidemiology of candidemia: 3-Year results from the emerging infections and the epidemiology of Iowa organisms study," Journal of Clinical Microbiology, vol. 40, no. 4, pp. 1298-1302, 2002.

[24] M. Cuenca-Estrella, L. Rodero, G. García-Effrón, and J. L. Rodriguez-Tudela, "Antifungal susceptibilities of Candida spp. isolated from blood in Spain and Argentina, 1996-1999," Journal of Antimicrobial Chemotherapy, vol. 49, no. 6, pp. 981-987, 2002.

[25] F. Sabatelli, R. Patel, P. A. Mann et al., "In vitro activities of posaconazole, fluconazole, itraconazole, voriconazole, and amphotericin B against a large collection of clinically important molds and yeasts," Antimicrobial Agents and Chemotherapy, vol. 50, no. 6, pp. 2009-2015, 2006.

[26] E. Skrodeniene, A. Dambrauskiene, and A. Vitkauskiene, "Susceptibility of yeasts to antifungal agents in Kaunas University of Medicine Hospital," Medicina, vol. 42, no. 4, pp. 294-299, 2006.

[27] M. Sojakova, D. Liptajova, M. Borovsky, and J. Subik, "Fluconazole and itraconazole susceptibility of vaginal yeast isolates from Slovakia," Mycopathologia, vol. 157, no. 2, pp. 163-169, 2004.

[28] A. S. Kantarcioglu and A. Yücel, "The species distrubition and antifungal susceptibility pattern of yeasts and molds isolated in the Department of Microbiology and Clinical Microbiology Deep Mycoses Laboratory between 01 April 1999-27 March 2001," Cerrahpasa Journal of Medicine, vol. 33, pp. 7-19, 2002.

[29] D. Kaya, S. Kaptanoğlu, Z. Üstüner, and O. Ertör, “Typing of yeasts isolated from the specimens of neutropenic patients and investigation of fluconazole resistance," Klimik Dergisi, vol. 14, no. 1, pp. 14-16, 2001.

[30] R. Kebudi, "New antifungal agents," Ankem Dergisi, vol. 21, no. 2, pp. 210-215, 2007.

[31] A. Espinel-Ingroff, E. Canton, D. Gibbs, and A. Wang, "Correlation of Neo-Sensitabs tablet diffusion assay results on three different agar media with CLSI broth microdilution M27-A2 and disk diffusion M44-A results for testing susceptibilities of Candida spp. and Cryptococcus neoformans to amphotericin B, caspofungin, fluconazole, itraconazole, and voriconazole," Journal of Clinical Microbiology, vol. 45, no. 3, pp. 858-864, 2007.

[32] B. D. Alexander, T. C. Byrne, K. L. Smith et al., "Comparative evaluation of Etest and Sensititre Yeast One panels against the Clinical and Laboratory Standards Institute M27-A2 reference broth microdilution method for testing Candida susceptibility to seven antifungal agents," Journal of Clinical Microbiology, vol. 45, no. 3, pp. 698-706, 2007.

[33] S. Keçeli Özcan, H. Ağırbaşlı, S. Çalışkan, and A. Willke, "Invitro susceptibilities of nonalbicans Candida and Yeasts other tan Candida Species isolated from patients with hematologic malignancies to fluconazole and voriconazole," Ankem Dergisi, vol. 21, no. 4, pp. 223-227, 2007.
[34] F. Aydin, G. Bayramoglu, N. C. Guler, N. Kaklikkaya, and I. Tosun, "Bloodstream yeast infections in a university hospital in Northeast Turkey: a 4-year survey," Medical Mycology, vol. 49, no. 3, pp. 316-319, 2011.

[35] M. A. Pfaller, L. Boyken, R. J. Hollis, S. A. Messer, S. Tendolkar, and D. J. Diekema, "In vitro susceptibilities of Candida spp. to caspofungin: four years of global surveillance," Journal of Clinical Microbiology, vol. 44, no. 3, pp. 760-763, 2006. 

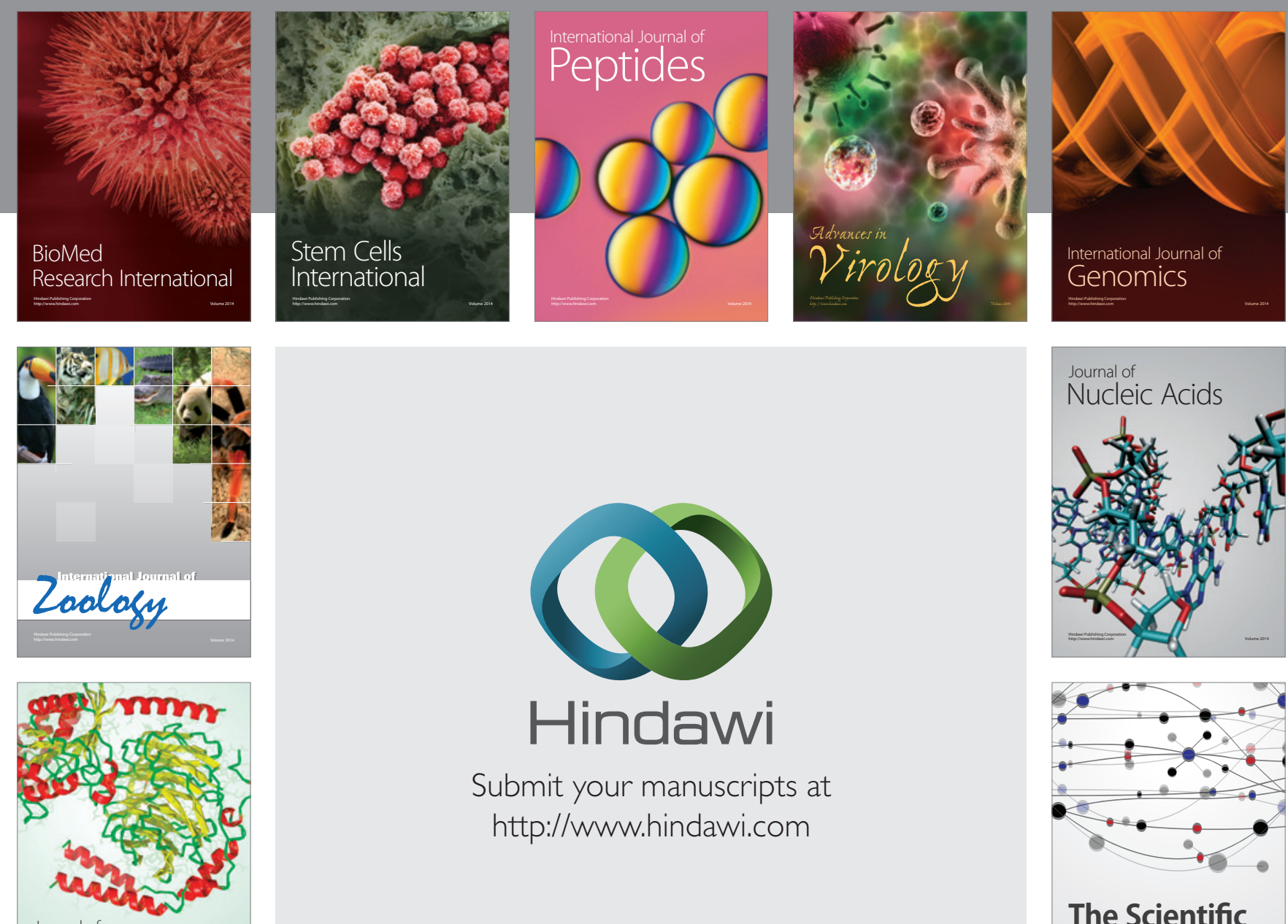

Submit your manuscripts at

http://www.hindawi.com

Journal of
Signal Transduction
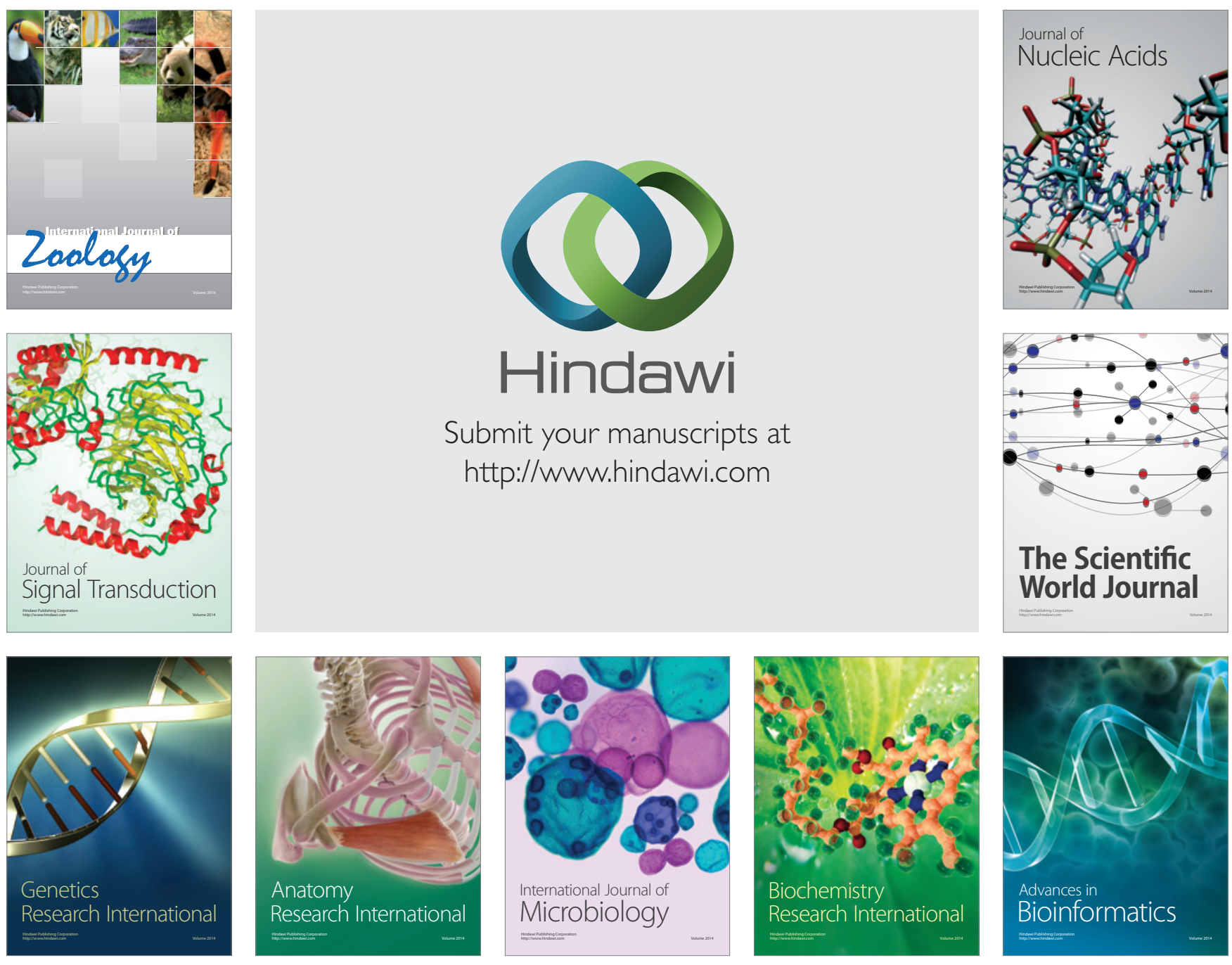

The Scientific World Journal
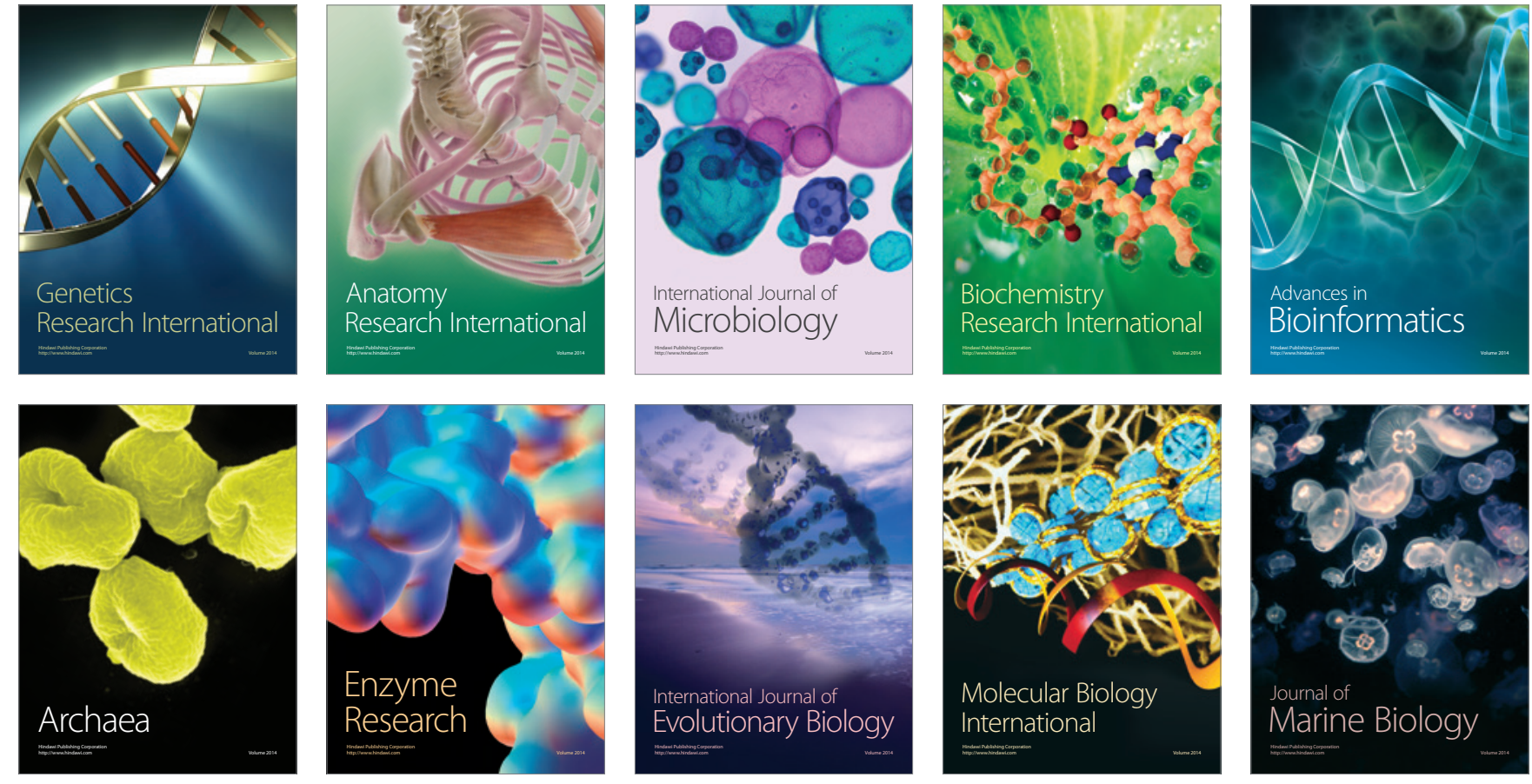